\title{
2D inversion of 3D magnetotelluric data: The Kayabe dataset
}

\author{
Xavier Garcia ${ }^{1}$, Juanjo Ledo ${ }^{1,2}$, and Pilar Queralt ${ }^{2}$ \\ ${ }^{1}$ Geological Survey of Canada, 615 Booth Street, Ottawa, Ontario, K1A 0E9, Canada \\ ${ }^{2}$ Departament de Geodinàmica i Geofísica, Universitat de Barcelona, Spain
}

(Received November 9, 1998; Revised May 13, 1999; Accepted May 17, 1999)

\begin{abstract}
In the last two Magnetotelluric Data Interpretation Workshops (MT-DIW) the participants were asked to model the Kayabe magnetotelluric dataset, a dense $(100 \mathrm{~m})$ grid of thirteen lines, with thirteen stations in each line. Bahr's phase-sensitive skew and the Groom and Bailey decomposition were used to select those lines for which the data could be considered two-dimensional. For these lines we used a 2D inversion algorithm to obtain a series of resistivity models for the earth. Finally, we constructed a 3D model using the 2D models and critically examined the validity and practicality of this approach based on 3D model study. We found that in the Kayabe dataset case the common practice of using 2D models to depict 3D models, can only be used to create a starting model for 3D interpretation. The sequential 2D models as a representation of a 3D body is unacceptable in terms of fit to the observed data. We question the validity of some of the conductivity structures in the 2D models, as they can be mere artifacts created by the algorithm to match $3 \mathrm{D}$ effects.
\end{abstract}

\section{Introduction}

The main objective of MT-DIW3 and MT-DIW4 was to focus attention on the modelling of MT data acquired in complex geological environments, in particular the Kayabe geothermal dataset. The Kayabe dataset consists of 209 magnetotelluric stations, 161 of which were acquired on a rectangular $100 \mathrm{~m}$ grid (Fig. 1). The New Energy and Industrial Technology Development Organization (NEDO) conducted this survey in order to have a better knowledge of the nearsurface geothermal resources of this area in the southern part of Hokkaido, Japan. A description of the data acquisition parameters and processing can be found in Takasugi (1992; and references therein). This area had been subjected to detailed geological and geophysical studies (Takasugi et al., 1992). The analysis and modelling of magnetotelluric data from this dense grid thus provides the opportunity to check the validity of the usual working hypothesis when information from fewer sites is available. At the same time we can gain insight into the validity of $2 \mathrm{D}$ interpretation of $3 \mathrm{D}$ bodies, at least for this particular case. Here we focus our efforts on interpretation of the data acquired on the grid. The grid has been divided into lines oriented perpendicular to the expected strike direction $(\mathrm{N} 30 \mathrm{~W})$ and indicated by the letters A-G (Fig. 1). Profiles oriented along strike are numbered from 0 to 12 .

\section{Decomposition}

MT data often contain the galvanic response of small near-surface heterogeneities that can distort and mask the response of regional structures (Jones, 1988; Torres-Verdin and Bostick, 1992). To detect and remove galvanic distortion

Copy right (C) The Society of Geomagnetism and Earth, Planetary and Space Sciences (SGEPSS); The Seismological Society of Japan; The Volcanological Society of Japan; The Geodetic Society of Japan; The Japanese Society for Planetary Sciences. effects, several decomposition methods have been devised to retrieve the regional information (Bahr, 1988; Jones, 1988; Groom and Bailey, 1989; Ledo et al., 1998). These methods essentially determine the dimensionality of the regional structures. As an initial step, the phase-sensitive skew (Bahr, 1988) was calculated for all sites over the entire period range of the data (Fig. 2). If the phase-sensitive skew is larger than 0.3 then the structure can be considered 3D (Bahr, 1988). In the current study, values smaller than 0.3 were obtained and thus we initially assume that the data represent the response of a 2D structure. As can be seen in Fig. 2, most of the stations show a phase-sensitive skew below 0.3 in the frequency range $1-100 \mathrm{~Hz}$. As the data outside of this range show erratic behavior and large skew values, we limit our interpretation to data from within this frequency range. To obtain the strike of the geoelectrical structures imaged in this period range, a multisite/multifrequency algorithm (McNeice and Jones, 1996) based on the Groom and Bailey (GB) decomposition method (Groom and Bailey, 1989) has been used.

Figure 3 shows the best regional strike azimuth determined for each site in the frequency range 100-1 Hz. The length of the arrows is inversely proportional to the fit of the data to the GB model. Short arrows means either that the decomposition model is inappropriate or the data errors are underestimated (Chave and Jones, 1997). The two longest arrows mean that the data are consistent with the model. Although the fit of the data to the GB models is not always acceptable, the strike is consistent between sites. As most of the lines did not show much larger misfit than when fixing the strike angle, we decided to leave the data in the original acquisition coordinates. Thus, the strike angle has been fixed to $\mathrm{N} 30 \mathrm{~W}$. The validity of this assumption will be checked later by calculating the response for the 3D model. Only those profiles having GB decomposition misfits within the confidence limits (95\% er- 


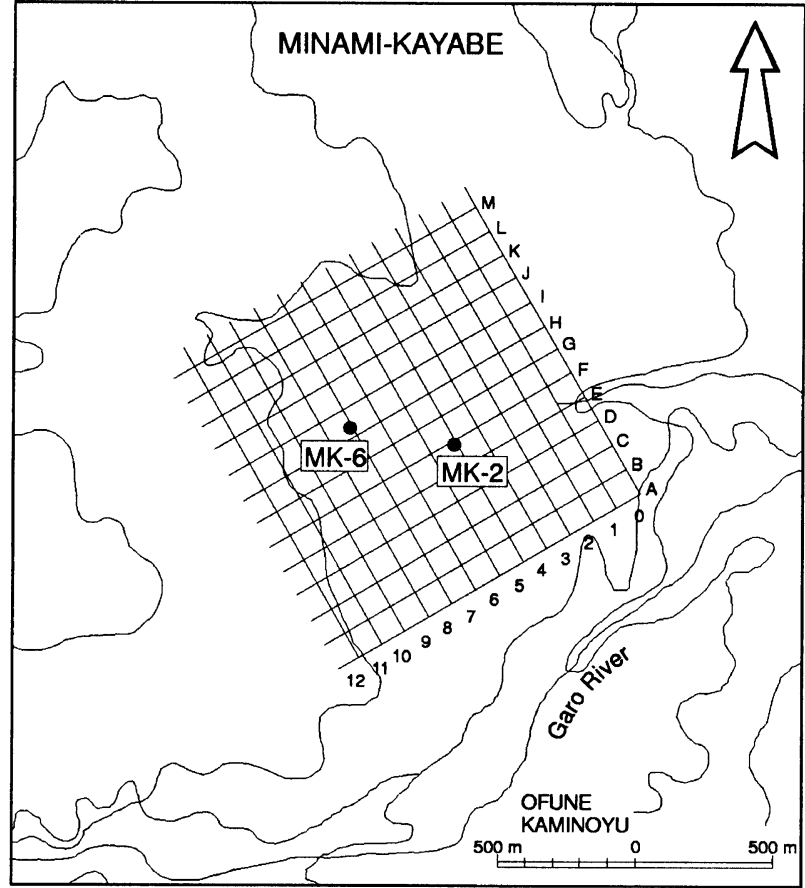

Fig. 1. Location of the area of study (Redrawn from Takasugi, 1992).

ror chi-2 distribution) for the majority of their stations, have been used to obtain $2 \mathrm{D}$ models. The profiles that can be modelled using 2D algorithms are: A, C, D, G, I, K, L, M, 1 and 6 . These profiles have been corrected for the twist and shear distortion prior to any modelling. Although the latter is not necessary for 2D interpretation, it can help in the subsequent $3 \mathrm{D}$ modelling as all the inductive and galvanic effects (except one multiplicative static shift factor) will be caused by the regional 3D structure.

\section{2D Inversion Models}

Aside from correction of the twist and shear distortion parameters, the data scatter is minor, and no further leveling of the measured data was done. To obtain resistivity models we used an inversion algorithm based on finding Tikhonov regularized solutions to the two-dimensional inverse problem for magnetotelluric data (Mackie, personal communication). Apparent resistivities and phases for both modes were inverted jointly to obtain the final models. Inversions were started with a trial model of a 1,000 Ohm.m homogeneous halfspace, and the best model obtained was used as a starting model for a new iteration. The statics have been inverted together with the model parameters. The grid used consisted in 160 horizontal elements, situated 5 equally distributed between neighboring stations and the rest for the extended side boundaries. Vertically, the grid varied between 20 and 25 elements.

Figure 4 shows the final inversion models obtained for the lines considered two-dimensional. The fit of the 2D model response to the data is generally acceptable with the normalized RMS misfit at a 5\% error floor in the range 2.3 to 5.9 (see Fig. 4, for details). Figure 5 shows a comparison between the model response and observed data for selected sites, chosen for a later contrast with the 3D model response.
One large shallow conductor is found on the eastern part of the profiles, extending from profile A to G (Figs. 3, 4(a) and 4(b)). This body is deeper on line I, and then continues north until line $M$. From line $A$ to $G$, this conductor lies beneath the lines 0 and 1 . Beneath line I, the conductor seems to extends to the south, beneath line 2, returning beneath line 1 in line $\mathrm{K}$. Along line $\mathrm{L}$, the conductor twists to the south again, beneath line 3 , returning to beneath line 1 in line M. The resistivity of this conductor is below $1 \mathrm{Ohm} . \mathrm{m}$, and its dimensions seems to change from line to line. The perpendicular profile 1 , helps to determine the continuation of the conductor between lines, providing a cross section of it.

Line 6 shows another strong conductor to the south of the profile. This conductor is bound by line A and C. Unfortunately, line B was one of the lines in which the GB decomposition failed, and this line could have helped to better determine the extension and conductivity of this body.

The last large anomaly observed in those models is along the southwestern part of the profiles A to G. Along the southwestern part of line I there is a shallow conductor, of small dimensions, and we don't believe that is related to the one observed under the rest of the profiles. Profiles K, L and $\mathrm{M}$ also show a conductor to the southwest, but it is difficult to relate because the fit of the model to the data is poor due to strong $3 \mathrm{D}$ effects for those southwestern stations.

All of these conductors are laterally thin (no more than $200 \mathrm{~m}$ ), and extend vertically from the near surface to 3 or $4 \mathrm{~km}$ depth. Beneath these conductors, we don't have more information as the maximum penetration depth is limited by the lowest frequency used $(1 \mathrm{~Hz})$.

Displayed in Fig. 6 are three 2D models, one of the NESW profiles (line G) and the two NW-SE profiles (lines 1 and 6) perpendicular to the first one, which provide a threedimensional perspective of the geoelectrical structures. The agreement of these profiles at the intersections is quite good, suggesting that this procedure can be used to obtain a starting 3D model. Still, these profiles cannot display all the information about the 3D structure and the use of the remainder of the NE-SW profiles is required to better track the conductive structures.

\section{3D Model and Discussions}

To determine the quality of the $2 \mathrm{D}$ interpretation of the data, a 3D model was constructed by interpolating between the available 2D NE-SW models. Although the main structures of the 2D models can be correlated along strike, the $3 \mathrm{D}$ model results in a complex structure. The orientation of the 3-D model is N30 W for the $x$-axis and $60 \mathrm{~N}$ for the $y$-axis, therefore the NW-SE lines correspond to the lines of constant $y$ and the NE-SW lines to lines with constant $x$. Figure 7 presents a schematic plan view of the 3D model corresponding to a depth of $1.5 \mathrm{~km}$ that sections the main conductive structures, that for simplicity of representation has its grid reduced to 1 element for each station. The mesh of the model is 99 by 99 horizontal elements and 22 vertical elements. The horizontal elements located at the core of the model (corresponding to the dense network MT sites) are squares of $25 \mathrm{~m}$, with elements of $12 \mathrm{~m}$ around resistivity boundaries. The response of the model has been calculated 


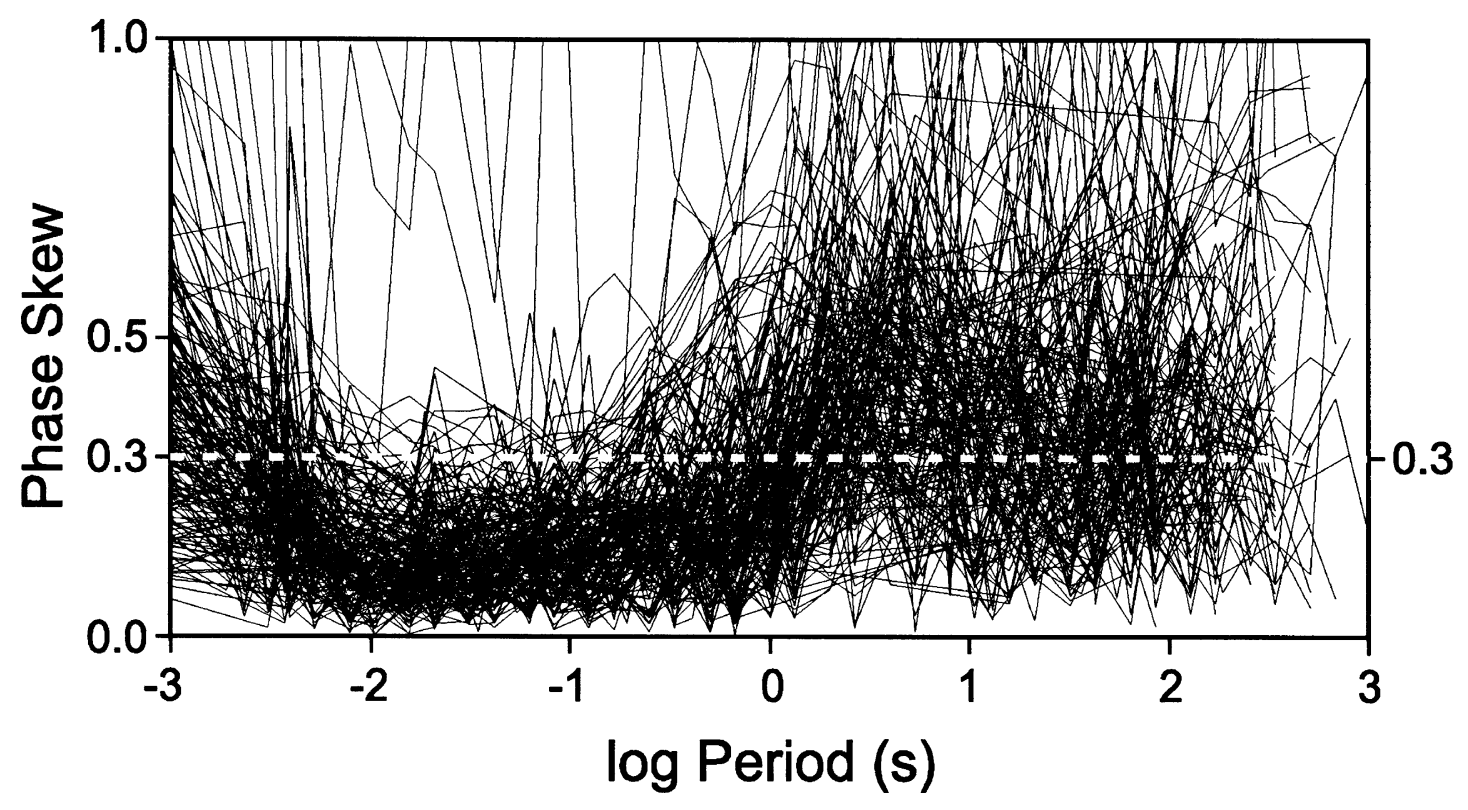

Fig. 2. Phase-sensitive skew calculated for all the sites and all the available frequencies. White dotted line corresponds to the 0.3 value, assumed as limit for $2 \mathrm{D}$ interpretation.

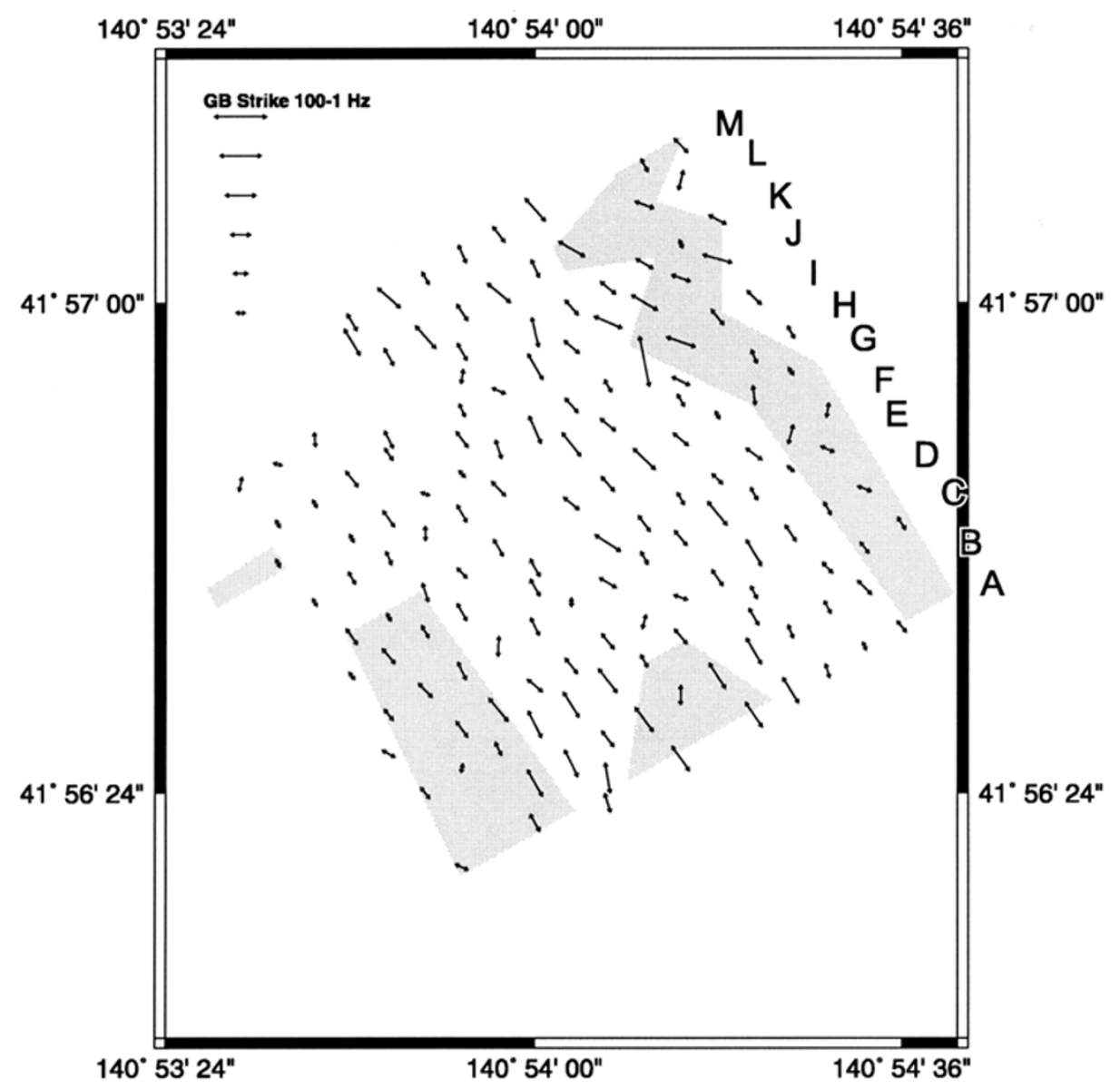

Fig. 3. GB strike obtained from multifrequency decomposition for the range 1-100 Hz. Length of the arrows is proportional to the fit. The two longest arrows indicate that the data fit the GB decomposition model, and the rest of the arrows indicate either the model is inappropriate or the data errors are too small. 
(a)

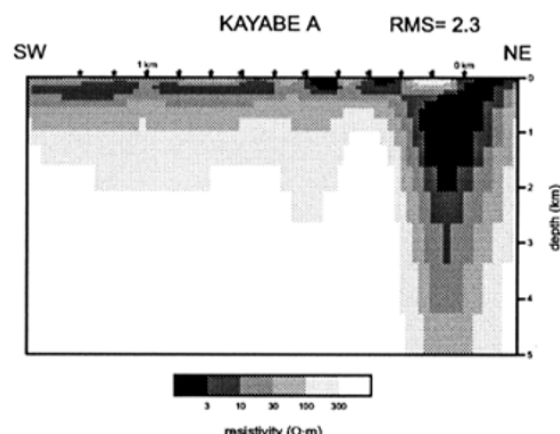

KAYABE D $\quad R M S=4.3$

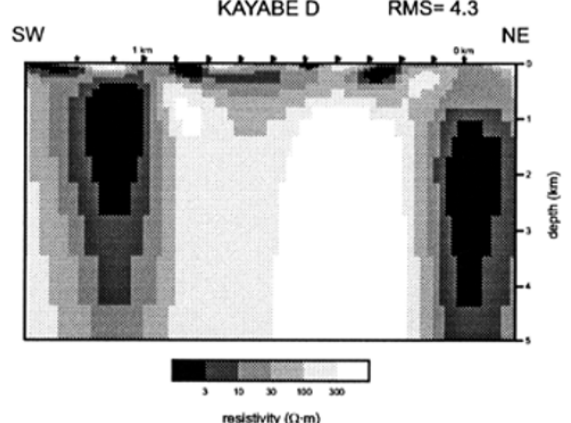

KAYABE I

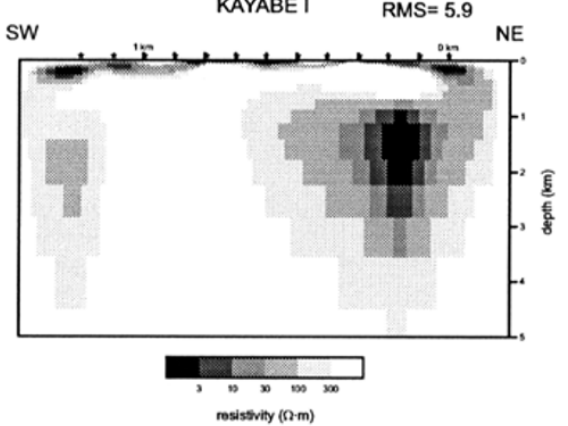

KAYABE L $\quad$ RMS $=2.6$

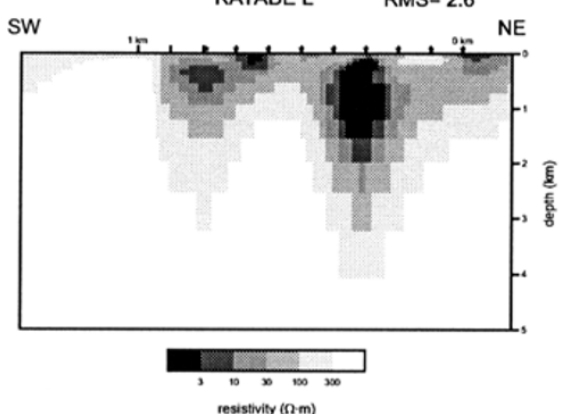

(b)

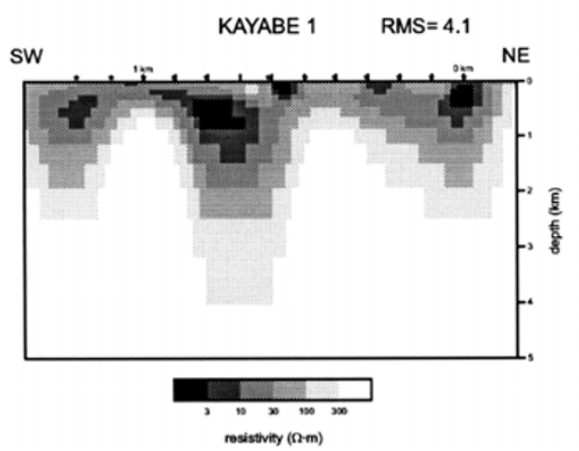

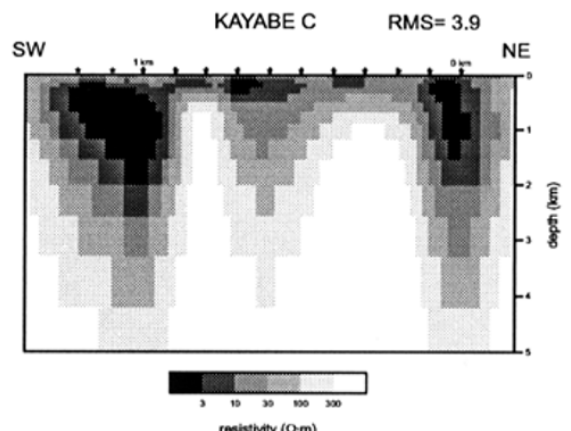

KAYABE G
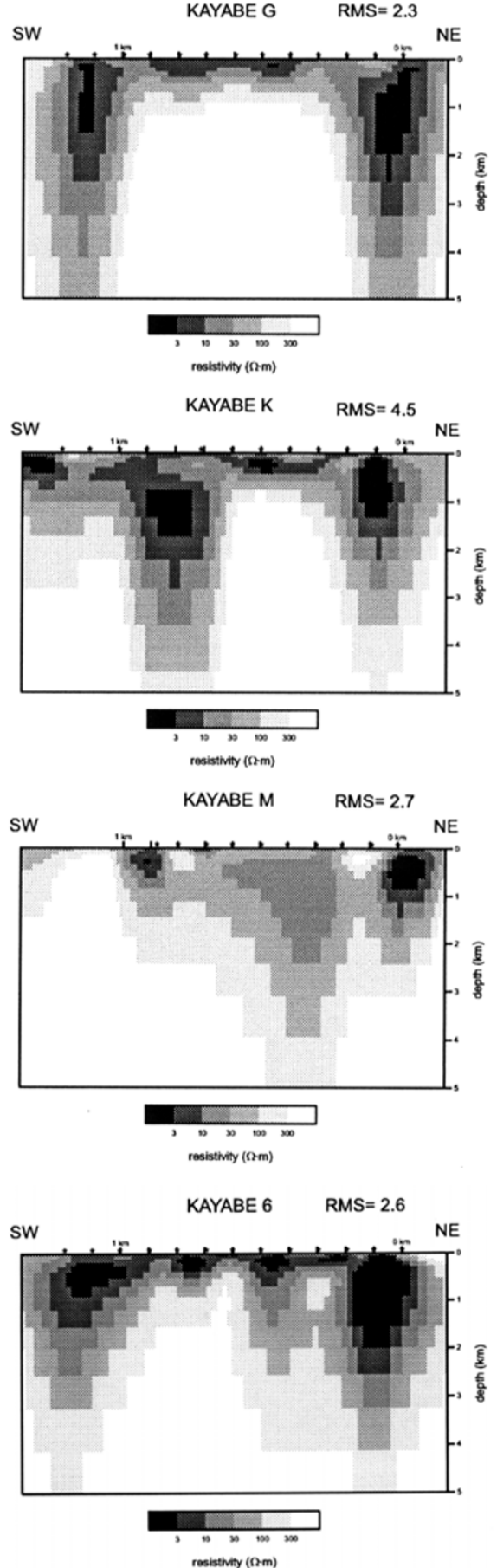

Fig. 4. 2D models obtained from the apparent resistivity and phase inversion of both polarizations for the frequency range 1-100 Hz. Normalized RMS misfit for the lines are as follows: Line A: 2.3; line C: 3.9; line D: 4.3; line G: 2.3; line I: 5.9; line K:4.5; line L: 2.6; line M: 2.7; line 1: 4.1 and line 6: 2.6. The stars show the location of the MT stations. The horizontal scale is shown by the position of the first station $(0 \mathrm{~km})$, and the $1 \mathrm{~km}$ tick, also $100 \mathrm{~m}$ ticks are shown. 

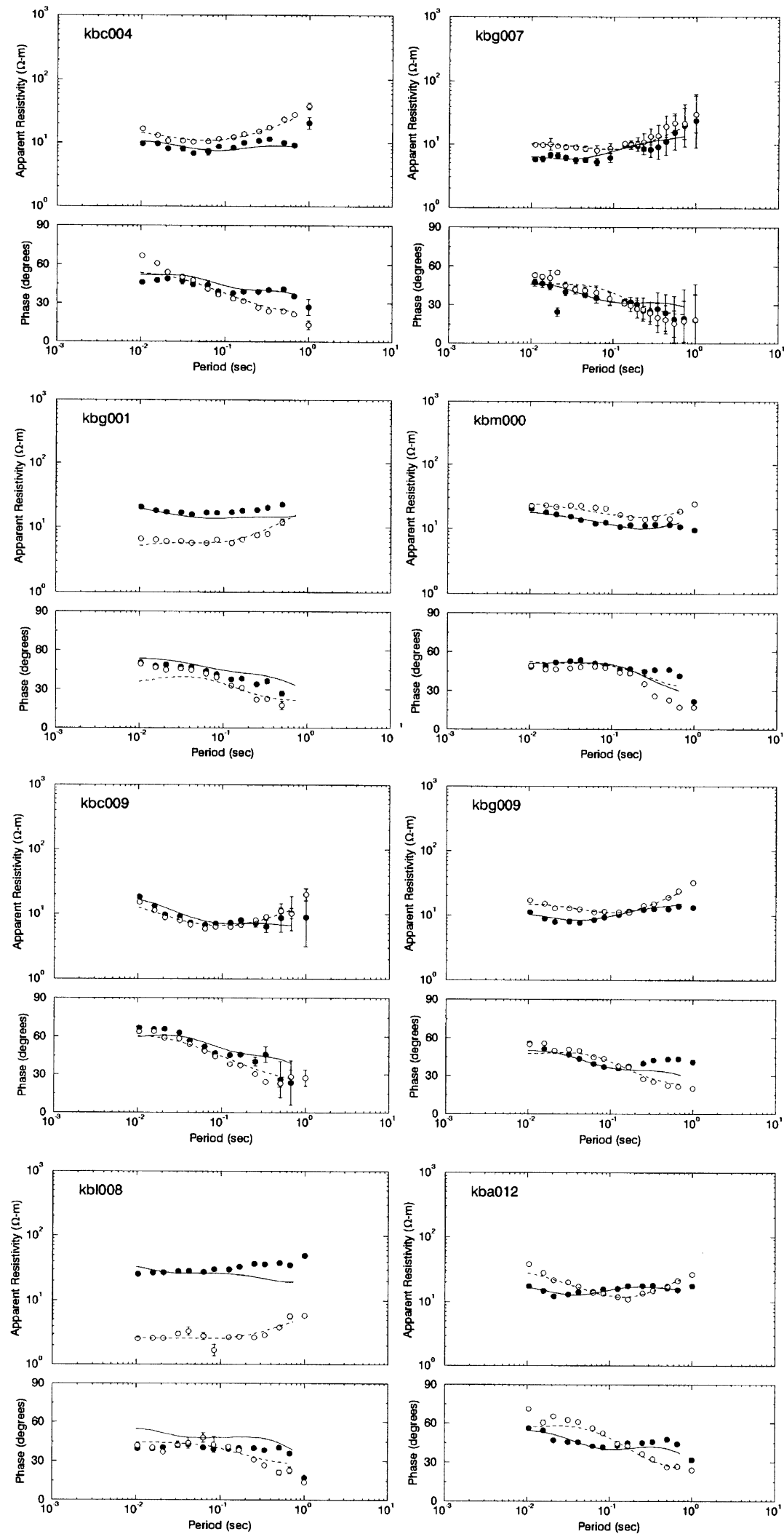

Fig. 5. Comparison of 2D model response and observed data for selected sites. Observed data: white circles ( $E$-Polarization), black circles ( $H$-Polarization), Synthetic data: solid line ( $E$-Polarization), dashed line ( $H$-Polarization). 


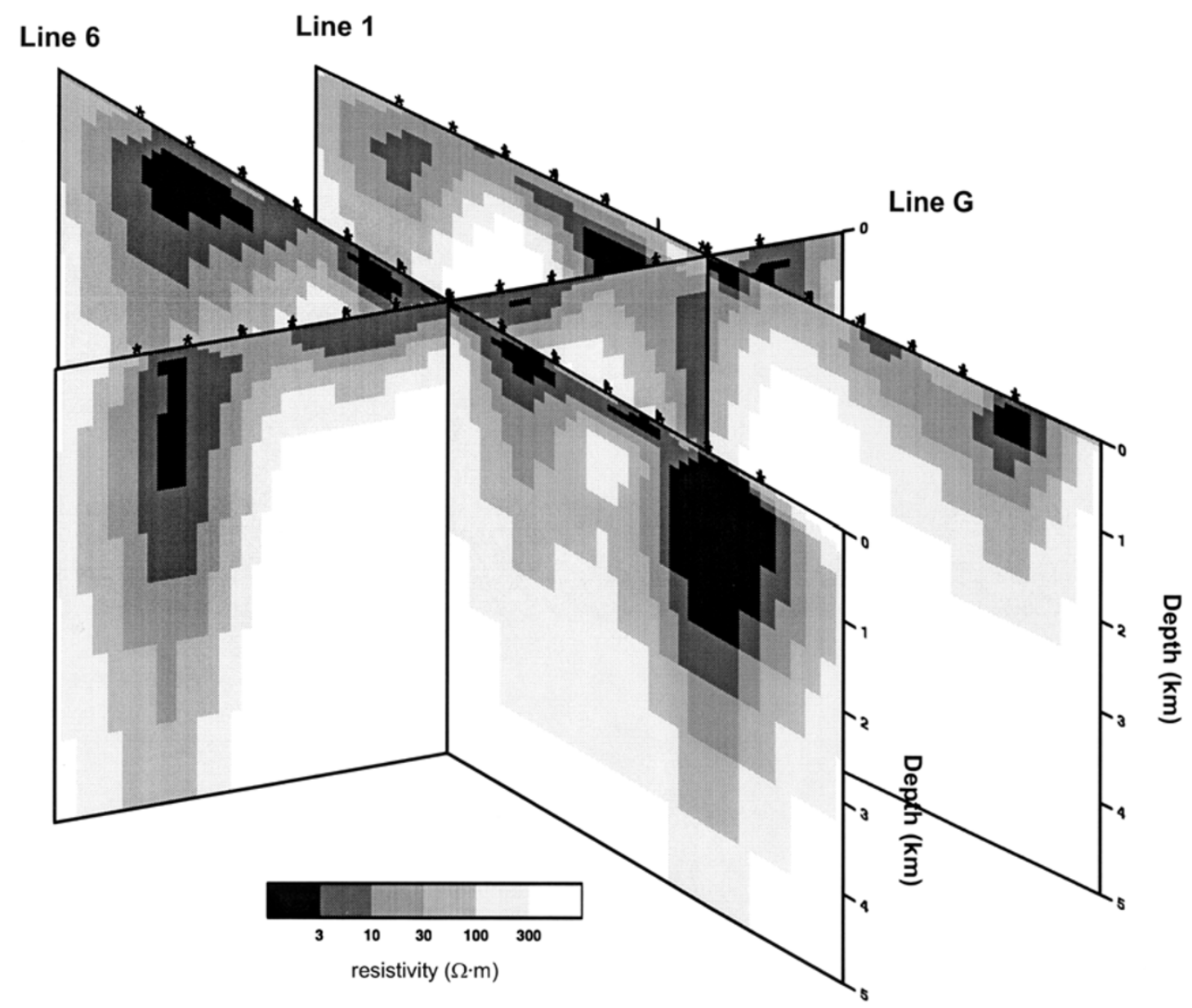

Fig. 6. Three-dimensional section of the area of study, obtained using three 2D models. The stars show the location of the MT sites and the horizontal ticks are every $100 \mathrm{~m}$.

using the code of Mackie et al. (1994) for seven frequencies in the frequency range $100-1 \mathrm{~Hz}$. To validate the numerical convergence of the 3D model we calculate the responses for the $3 \mathrm{D}$ model using different mesh grids. For the finest mesh used $(99 \times 99 \times 22)$ the 3 D responses show a stable behavior, with differences from the other models tested of less than the error level of the data. As an example we show in Fig. 8 the different responses obtained for station $\mathrm{G} 7$, representative of the results achieved for the whole dataset.

The fit of the data is not as good as required, but is admissible as a preliminary 3D model, remembering that only information from the impedance tensor has been considered, not using the transfer functions of the vertical magnetic field. Figure 9 shows the comparison between the 3D model response and observed data of eight selected sites (same sites as Fig. 5). The response of sites $\mathrm{c} 4, \mathrm{~g} 1, \mathrm{~g} 7$ and $\mathrm{m} 0$ fit the data reasonably well; these sites are located in the central and east area of the network (see Fig. 7). The sites located in the west, c9, g9, 18 and a12, show a large misfit. This fact can be related to the finite strike length of the structures located in the west. In the eastern area the main structures are continuous along strike. In the western area, large conductors that cut the more resistive structure along the strike direction cause a lateral current channeling effect. The validity of a 2D interpretation of 3D structures will depend on the relationship between the frequency of interest, size of the anomalous structure and distance to it.

From these results, the hypothesis of bidimensionality was not valid for all the profiles that have been inverted, even if the phase-sensitive skew was below the 0.3 limit and a good fit of the 2D model was reached. The question that naturally arises is: which of the structures presented in the $2 \mathrm{D}$ model can be considered as real and which are artifacts due to the error of interpreting 3D structures as 2D? It seems from the data fit that lateral variations along strike in the $3 \mathrm{D}$ model are overestimated. In our case it corresponds to the structures located in the west. However the conductive structure located below lines 0 and 1 has more credibility.

\section{Conclusions}

The Kayabe dataset has been very useful in showing the possibilities and limits of the 2D interpretation of 3D structures. This is a very particular case in which some general ideas about MT modelling in complex areas can be obtained. In this case, it seems that a phase-sensitive skew smaller than 0.3 doesn't means necessarily that the data is not $3 \mathrm{D}$. The multifrequency GB decomposition for each site and the multisite/multifrequency GB decomposition for some lines have allowed us to retrieve the strike of the main structures even in the presence of $3 \mathrm{D}$ bodies. The $2 \mathrm{D}$ inversion of these 


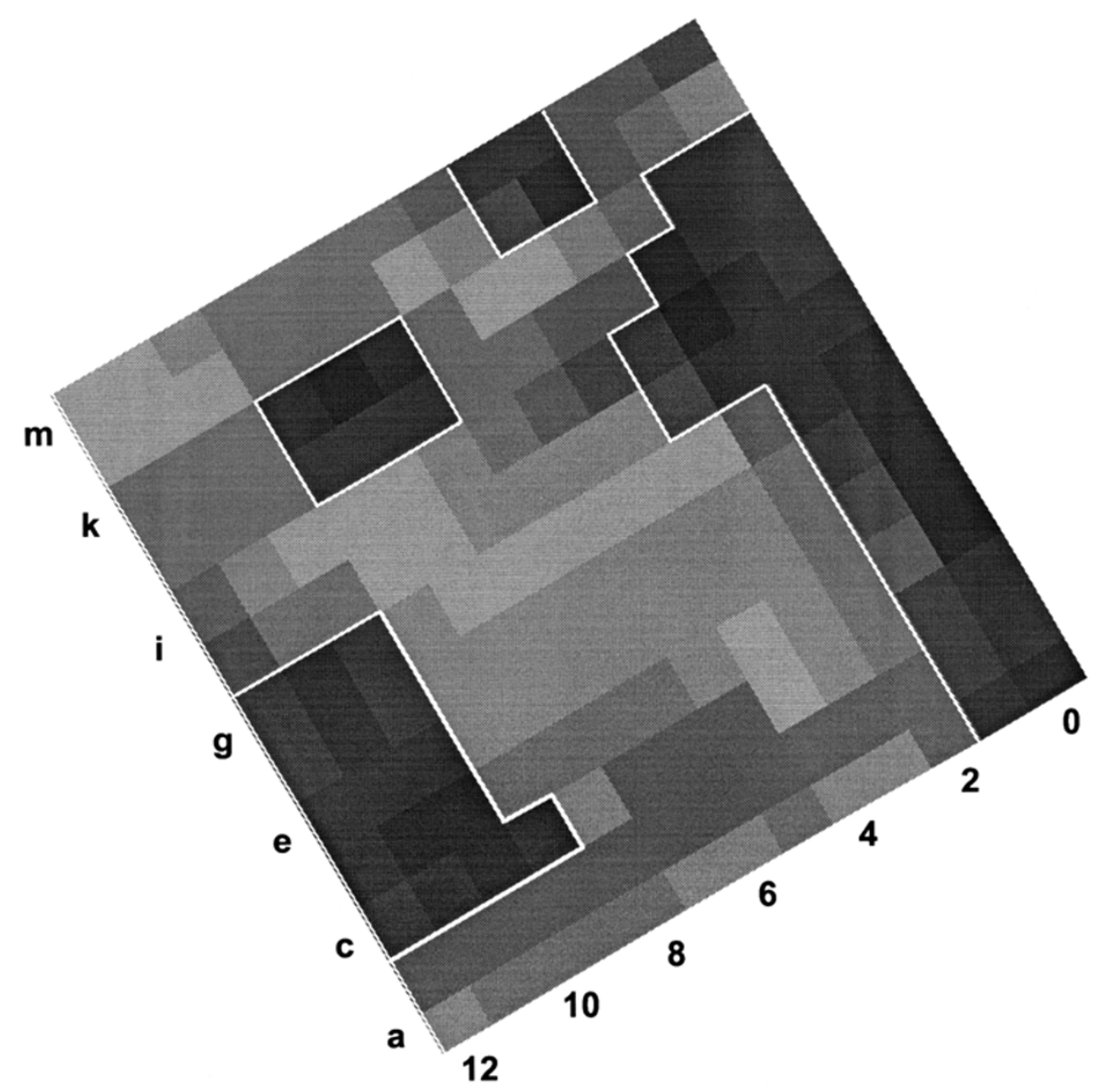

Fig. 7. Schematic plan view of the 3D model at $1.5 \mathrm{~km}$ depth using a $100 \mathrm{~m}$ grid representative of the bulk resistivity, the 3D model used in the calculations consisted in a $99 \times 99$ elements grid. Outlined areas correspond to resistivity lower than $100 \mathrm{Ohm} . \mathrm{m}$.

a)
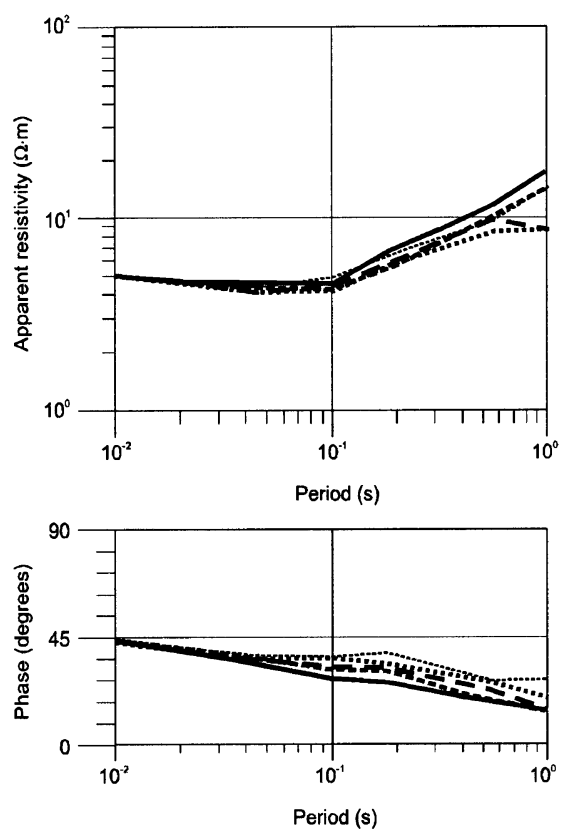

b)

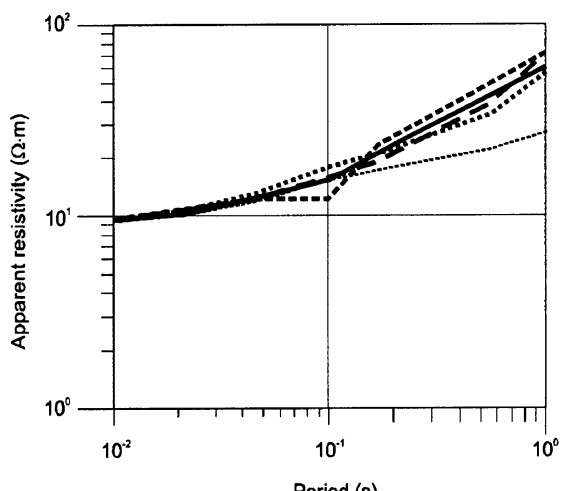

Period (s)

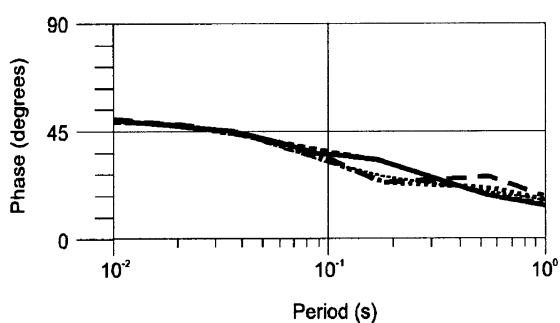

Fig. 8. Convergence analysis of the 3D model using different mesh grids for station G7 (kbg007). a) Apparent resistivities and phases $X Y$. b) Apparent resistivities and phases $Y X$. Solid line: $99 \times 99 \times 22$ grid elements. Short dashed line: $74 \times 76 \times 22$. Long dashed line: $57 \times 61 \times 22$. Dotted line: $32 \times 32 \times 22$. Thin dotted line: $19 \times 19 \times 22$. 

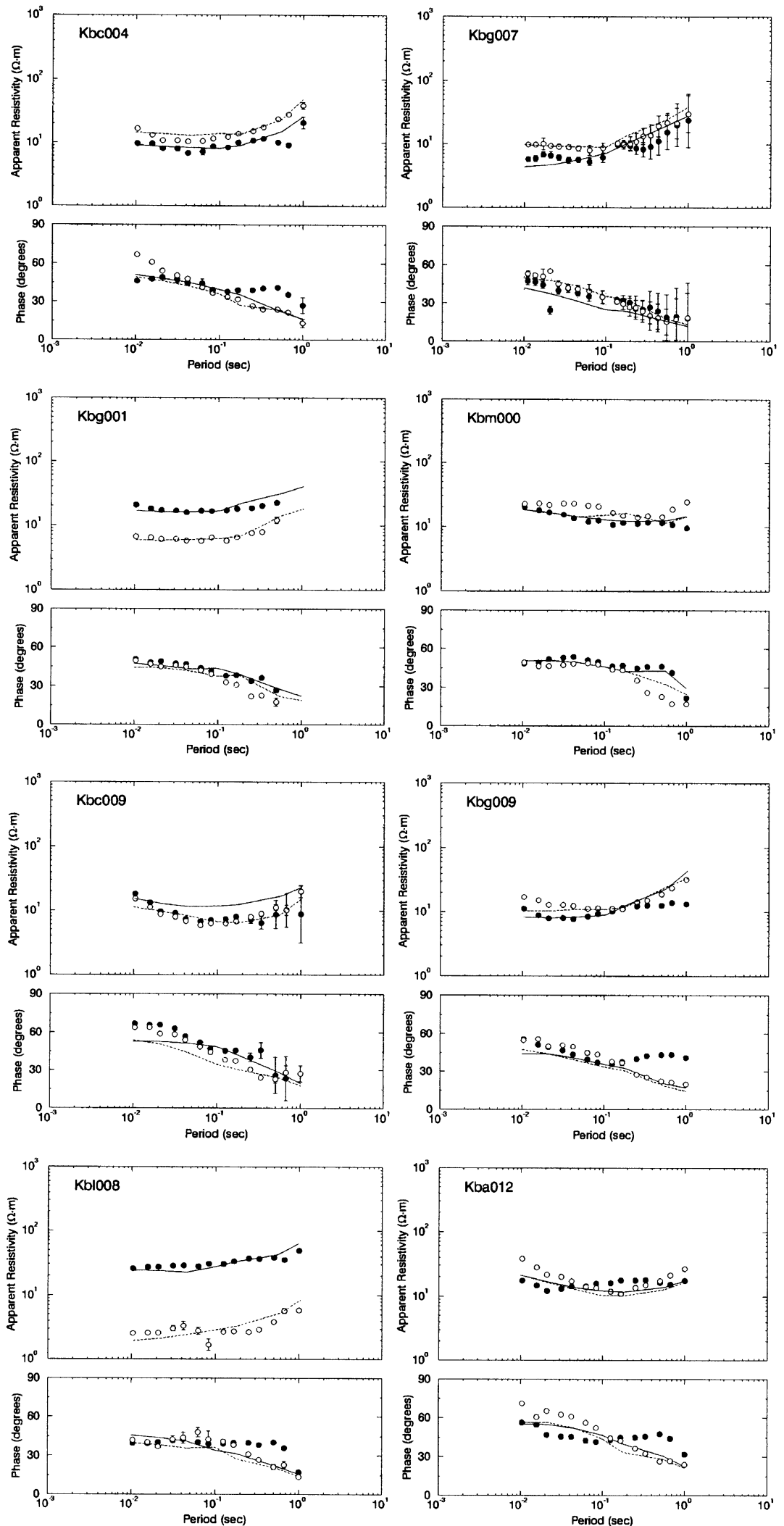

Fig. 9. Comparison of 3D model response and observed data for selected sites. Observed data: white circles ( $x y$-Polarization), black circles ( $y x$-Polarization), Synthetic data: solid line ( $x y$-Polarization), dashed line ( $y x$-Polarization). 
profiles gives us a first approach to the electrical structure with a good data fit for all the sites along the lines. However, when a $3 \mathrm{D}$ model was created using the $2 \mathrm{D}$ models, the misfit presents an important bias and it is not uniformly distributed. The sites located near finite strike structures have larger misfits. This result must be related to lateral current channeling effects, due to the lateral finite extension of the structures. The current channeling introduces false structures in the 2D models. Although not all the available data have been used, the $3 \mathrm{D}$ model obtained from the $2 \mathrm{D}$ data inversion will be a useful starting model.

Acknowledgments. NEDO kindly allows us the use of this data. The authors would like to thank Alan G. Jones, David Boerner and Don White for the comments and suggestions. Two anonymous reviewers and John Weaver are also acknowledged. XG gratefully acknowledges the MT-DIW4 organization for the financial support to attend the workshop held in Sinaia. The attendance of PQ at the MT-DIW4 was supported by DGICYT of Spain, project PB950269. The attendance of JL was supported by a grant from the Spanish Ministry of Education. GSC contribution 1999048.

\section{References}

Bahr, K., Interpretation of the magnetotelluric impedance tensor: regional induction and local telluric distortion, J. Geophys., 62, 119-127, 1988.
Chave, A. and A. G. Jones, Electric and magnetic field galvanic distortion decomposition of BC87 data, J. Geomag. Geoelectr., 49, 767-789, 1997.

Groom, R. W. and R. C. Bailey, Decomposition of magnetotelluric impedance tensors in the presence of local three-dimensional galvanic distortions, J. Geophys. Res., 94, 1913-1925, 1989.

Jones, A. G., Static shift of magnetotelluric and its removal in a sedimentary environment, Geophysics, 43, 1157-1166, 1988.

Ledo, J. J., P. Queralt, and J. Pous, Effects of galvanic distortion on magnetotelluric data over a three-dimensional structures, Geophys. J. Int., 132, 295-301, 1998.

Mackie, R. L., J. T. Smith, and T. R. Madden, Three-dimensional electromagnetic modeling using finite difference equations: the magnetotelluric example, Radio Sci., 29(4), 923-935, 1994.

McNeice, G. and A. G. Jones, Multisite, multifrequency tensor decomposition of magnetotelluric data, in Society of Exploration Geophysicists 66th Annual Meeting, Expanded Abstracts, pp. 281-284, 1996.

Takasugi, S., Analyses of magnetotelluric fields for a three dimensional Earth on the basis of the transfer functions, J. Geomag. Geoelectr., 44, 325-344, 1992.

Takasugi, S., K. Tanaka, N. Kawakami, and S. Muramatsu, High spatial resolution of the resistivity structure revealed by a dense network MT measurements-A case study in the Minabikayabe area, Hokkaido, Japan, J. Geomag. Geoelectr., 44, 289-308, 1992.

Torres-Verdin, C. and F. X. Bostick, Implication of the Born approximation for the magnetotelluric problem in three-dimensional environments, Geophysics, 57, 587-602, 1992.

X. Garcia (e-mail: garcia@cg.nrcan.gc.ca), J. Ledo, and P. Queralt 